\title{
Stochastic Finite Element Framework for Cardiac Kinematics Function and Material Property Analysis
}

\author{
Pengcheng Shi and Huafeng Liu \\ Biomedical Research Laboratory \\ Department of Electrical and Electronic Engineering \\ Hong Kong University of Science and Technology \\ Clear Water Bay, Kowloon, Hong Kong \\ \{pengcheng.shi, eeliuhf\}@ust.hk
}

\begin{abstract}
A stochastic finite element method (SFEM) based framework is proposed for the simultaneous estimation of cardiac kinematics functions and material model parameters. While existing biomechanics studies of myocardial material constitutive laws have assumed known kinematics, and image analyses of cardiac kinematics have relied on chosen constraining models (mathematical or mechanical), we believe that a probabilistic strategy is needed to achieve robust and optimal estimates of kinematics functions and material parameters at the same time. For a particular a priori patient-dependent constraining material model with uncertain parameters and a posteriori noisy observations, stochastic differential equations are combined with the finite element method. The material parameters and the imaging/image-derived data are treated as random variables with known prior statistics in the dynamic system equations of the heart. In our current implementation, extended Kalman filter $(\mathrm{EKF})$ procedures are adopted to linearize the equations and to provide the joint estimates. Because of the periodic nature of the cardiac dynamics, we conclude experimentally that it is possible to adopt this physical-model based optimal estimation approach to achieve converged estimates. Results from canine MR phase contrast images with linear elastic model are presented.
\end{abstract}

\section{Introduction}

Myocardial dynamics can be stated by the following material parameter dependent differential equation:

$$
\Phi(q, u(q))=\Pi(u(q))
$$

with constraining mechanical model parameters $q$, kinematics states $u(q)$, system differential operators $\Phi$, and loads $\Pi$. With this dynamic system, finite element method (FEM) has been used as a natural framework for the biomechanics studies of myocardium material constitutive laws with observations/measurements on the kinematics states [4,6], and physically motivated image analyses of cardiac kinematics properties with assumed mechanical models [29]. 
In biomechanics studies, the kinematics of the heart tissues is assumed known from implanted markers or imaging means. The issue is then to use these kinematics observations to estimate material parameters of the constitutive laws. While most works deal with regional finite deformations measured at isolated locations [4, MR tagging has been used more recently for the in vivo study of the mechanics of the entire heart. In [6], unknown material parameters were determined for an exponential strain energy function that maximized the agreement between observed (from MR tagging) and predicted (from FEM modeling) regional wall strains. However, it is well recognized that the recovery of kinematics from MR tagging or other imaging techniques is not a solved problem yet, and constraining models of mechanical nature may be needed to for the kinematics recovery in the first place [2].

In image-based analyses of cardiac kinematics functions, assumptions must be made about the myocardial behavior in order to constrain the ill-posed problem for a unique solution [2]. These constraining models could be either mathematically motivated regularization such as in [7, or continuum mechanics based energy minimization such as in 9. Conjugate to biomechanics efforts, image analysis works are based on the premise that material or other model properties are known as prior information, and the issue is to use these models to estimate kinematics parameters in some optimal sense. The selection of an appropriate model with proper parameters largely determines the quality of the analysis results. Yet, for any given data, the precise or even reasonable material/mathematical models and parameters are usually not readily known $a$ priori.

In this paper, we present a stochastic finite element framework for the simultaneous estimation of the cardiac kinematics functions and the material constitutive parameters from image sequence. Given the uncertainty of the material properties for a particular patient and the noisy nature of the imaging data, we believe that a probabilistic strategy is needed to achieve robust and optimal estimates for a particular a priori constraining model with uncertain parameters and a posteriori noisy observations. Coupling stochastic modeling of the myocardium with finite element method, we can now deal with noisy imaging/imaging-derived data and uncertain constraining material parameters in a coordinated effort. Because of the periodic nature of the cardiac behavior, we will show experimentally that it is possible to adopt this physical model based statistical estimation approach to achieve converged estimates.

\section{Methodology}

\subsection{Stochastic Finite Element Method}

Stochastic finite element method (SFEM) has been used for structural dynamics analyses in probabilistic frameworks [1]. In SFEM, structural material properties are described by random fields, possibly with known prior statistics, and the observations and loads are corrupted by noises. Hence, stochastic differential dynamics equations are combined with the finite element method to study the dynamic structures with uncertainty in their parameters and measurements. 


\subsection{Stochastic Dynamic Equation}

For computational feasibility, our current implementation assumes temporally constant linear elasticity of the myocardium with varying spatial distributions of the Young's modulus and the Poisson's ratio. We derive the myocardial dynamics equation within a finite element framework to be:

$$
M \ddot{U}+C \dot{U}+K U=R
$$

with $M, C$ and $K$ the mass, damping and stiffness matrices, $R$ the load vector, and $U$ the displacement vector. $M$ is a known function of material density and is temporally and spatially constant. $K$ is a function of the material constitutive law (the strain-stress relationship), and is related to the material-specific Young's modulus $E$ and Poisson's ratio $\nu$, which can vary temporally and spatially. In our framework, these two local parameters are treated as random variables with known a priori statistics for any given data, and are needed to be estimated along with the kinematics functions (in this paper, we do not consider the temporal dependency of the material parameters). $C$ is frequency dependent, and we assume Rayleigh damping with $C=\alpha M+\beta K$. Equation (2) is a stochastic differential equation in nature per Ito's calculus.

\subsection{State Space Model}

The above dynamics equation can be transformed into a state-space representation of a continuous-time linear stochastic system:

$$
\dot{x}(t)=A_{c}(\theta) x(t)+B_{c} w(t)
$$

where the material parameter vector $\theta$, the state vector $x$, the system matrices $A_{c}$ and $B_{c}$, and the control (input) term $w$ are:

$$
\begin{aligned}
\theta & =\left[\begin{array}{c}
E \\
\nu
\end{array}\right], \quad x(t)=\left[\begin{array}{c}
U(t) \\
\dot{U}(t)
\end{array}\right], \quad w(t)=\left[\begin{array}{l}
0 \\
R
\end{array}\right], \\
A_{c} & =\left[\begin{array}{cc}
0 & I \\
-M^{-1} K & -M^{-1} C
\end{array}\right], \quad B_{c}=\left[\begin{array}{cc}
0 & 0 \\
0 & M^{-1}
\end{array}\right]
\end{aligned}
$$

Meanwhile, the observed imaging/imaging-derived data $y(t)$ can be expressed in the measurement equation:

$$
y(t)=D x(t)+e(t)
$$

where $D$ is a known measurement matrix, and $e(t)$ is the measurement noise which is additive, zero mean, and white $\left(E[e(t)]=0, E\left[e(t) e(s)^{\prime}\right]=R_{e}(t) \delta_{t s}\right)$.

In our case, Equations (3) and (4) describe a continuous-time system with discrete-time measurements (the imaging/imaging-derived data), or a so-called sampled data system. The input is computed from the system equation, and is piecewise constant over the sampling interval $T$. Thus, we arrive at the system equations [3]:

$$
\begin{gathered}
x((k+1) T)=A x(k T)+B w(k T) \\
A=e^{A_{c} T}, \quad B=A_{c}^{-1}\left(e^{A_{c} T}-I\right) B_{c}
\end{gathered}
$$



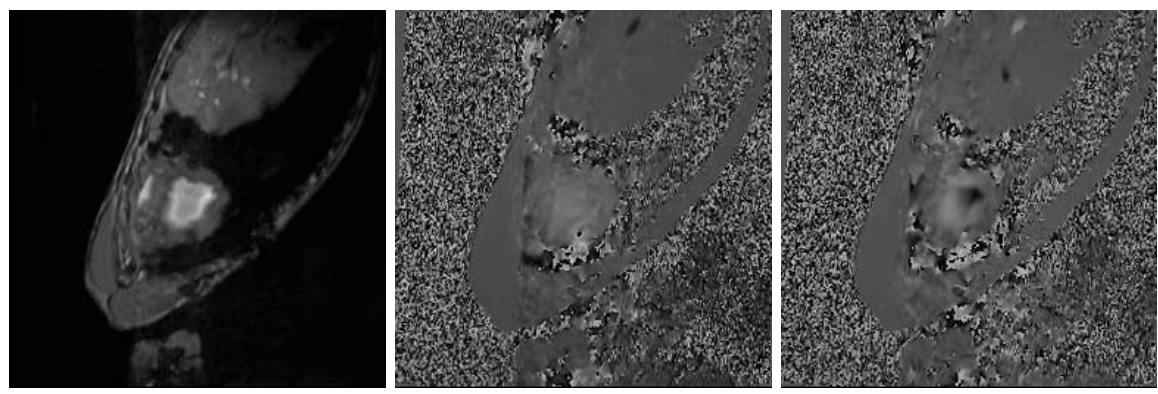

Fig. 1. Canine MR phase contrast images: magnitude (left), x-velocity (middle), and y-velocity (right).

For general continuous-time system with discrete-time measurements, including the additive, zero-mean, white process noise $v\left(E[v(t)]=0, E\left[v(t) v(s)^{\prime}\right]=\right.$ $Q_{v}(t) \delta_{t s}$, independent of $\left.e(t)\right)$, we have the following state equation:

$$
x(t+1)=A(\theta) x(t)+B(\theta) w(t)+v(t)
$$

\subsection{Extended Kalman Filter for Joint State and Parameter Identification}

We can then augment the state vector $x$ by the material parameter vector $\theta$ to

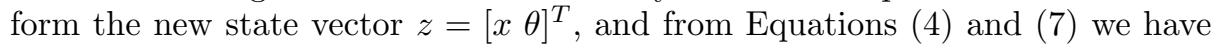
the following pair of augmented state/measurement equations:

$$
\begin{aligned}
z(t+1) & =\left[\begin{array}{c}
A(\theta) x(t)+B(\theta) w(t) \\
\theta
\end{array}\right]+\left[\begin{array}{c}
v(t) \\
0
\end{array}\right]=f(z(t), w(t))+\left[\begin{array}{c}
v(t) \\
0
\end{array}\right] \\
y(t) & =\left[\begin{array}{ll}
D & 0
\end{array}\right]\left[\begin{array}{c}
x(t) \\
\theta(t)
\end{array}\right]+\left[\begin{array}{c}
e(t) \\
0
\end{array}\right]=h(z(t))+\left[\begin{array}{c}
e(t) \\
0
\end{array}\right]
\end{aligned}
$$

The joint state and parameter estimation problem can be understood as a state estimation problem for a nonlinear system, and this form of formulation leads to a solution of the filtering problem based on continuous-system-discretemeasurement extended Kalman filter (EKF) framework, which is based on linearization of the augmented state equations at each time step. A recursive procedure with natural block structure is used to perform the joint state (kinematics) and parameter (material) estimation, and a general analysis of the convergence of the algorithm can be found in $[5]$ :

$$
\begin{aligned}
\hat{x}(t+1) & =A(\hat{\theta}(t)) \hat{x}(t)+B(\hat{\theta}(t)) w(t)+L(t)[y(t)-D \hat{x}(t)] \\
\hat{\theta}(t+1) & =\hat{\theta}(t)+G(t)[y(t)-D \hat{x}(t)] \\
\hat{x}(0) & =\hat{x}_{0}, \quad \hat{\theta}(0)=\hat{\theta}_{0}
\end{aligned}
$$

where

$$
L(t)=\left[A(\hat{\theta}(t)) P_{1}(t) D^{T}+M_{t} P_{2}^{T}(t) D^{T}\right] S^{-1}(t)
$$



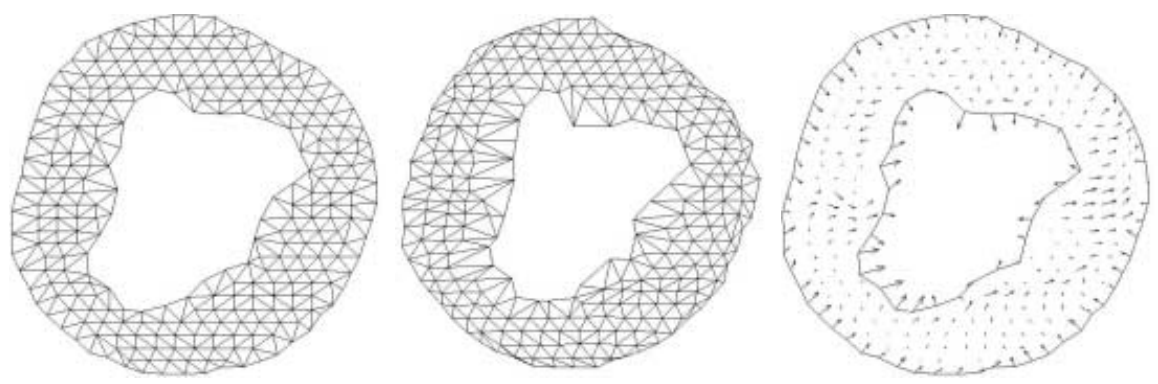

Fig. 2. Finite element meshes of the left ventricle: original (left, end-diastole), deformed (middle, end-systole), and ED to ES displacement map (right).

$$
\begin{aligned}
G(t)= & P_{2}^{T}(t) D^{T} S^{-1}(t) \\
P_{1}(t+1)= & A(\hat{\theta}(t))\left[P_{1}(t) A^{T}(\hat{\theta}(t))+P_{2}(t) M_{t}^{T}\right] \\
& +M_{t}\left[P_{2}^{T}(t) A^{T}(\theta(t))+P_{3}(t) M_{t}^{T}\right]+Q_{v}-L(t) S(t) L^{T}(t) \\
P_{2}(t+1)= & A(\hat{\theta}(t)) P_{2}(t)+M_{t} P_{3}(t)-L(t) S(t) G^{T}(t) \\
P_{3}(t+1)= & P_{3}(t)-G(t) S(t) G^{T}(t) \\
S(t)= & D P_{1}(t) D^{T}+R_{e} \\
M_{t}= & \left.\frac{\partial}{\partial \theta}(A(\theta) \hat{x}+B w(t))\right|_{\theta=\hat{\theta}} \\
P(0)= & {\left[\begin{array}{ll}
P_{1}(0) & P_{2}(0) \\
P_{2}^{T}(0) & P_{3}(0)
\end{array}\right] }
\end{aligned}
$$

\subsection{Computational Considerations}

In our current 2D implementation, the left ventricle is Delaunay triangulated from the sampled end-diastolic myocardial points. Using the linear elastic model with uncertain parameters, the stiffness, mass, damping, and load matrices for each element and the entire ventricle are constructed and the SFEM framework is formed. Imaging and imaging-derived data are incorporated as the initial and boundary conditions, and are used in the optimization process.

Initial conditions: the use of the EKF algorithm for joint state and parameter estimation requires initial values for both the augmented state vector and the augmented state error covariance matrix $P(0)$, whose values are proportional to the expected errors in the corresponding parameters to ensure smooth convergence. As pointed in [8], if the covariance matrix expresses the errors in $1 \sigma$ (stand deviation) format, then the actual errors of the estimates will be within $\pm 3 \sigma$. For accuracy and computation considerations, we set the initial values of the covariance matrix for the displacements as 0.1 , for the Poisson ratio as 0.001, and for the Young's modulus as 2000 in our current experiment. In addition, we model the process noise $Q_{v}$ and measurement noise $R_{e}$ as diagonal matrix, and for this paper we use fixed values for both. Specifically, let $i$ be the frame 

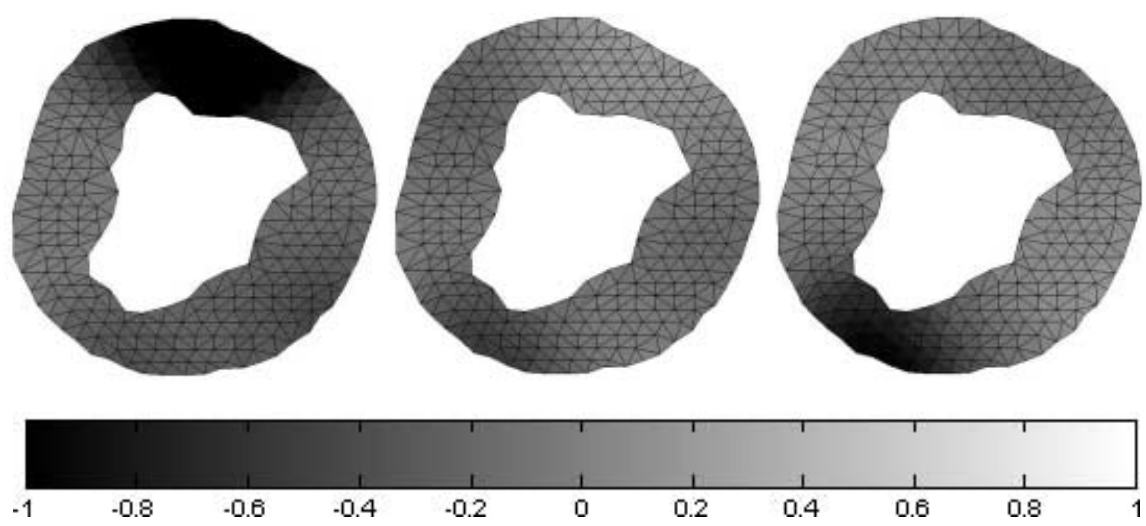

Fig. 3. Estimated $x$-strain (left), y-strain (middle), and shear strain (right) maps between ED and ES.

number, and $j$ be the loop number (because the periodic nature of the cardiac dynamics, we can loop through the image sequence until convergence):

- If $j=1$ and $i=1$, the initial displacements are zero. Otherwise, the initial displacements are the estimates from previous frames/loops up to $j^{\text {th }}$ loop, $(i-1)^{t h}$ frame.

- MR phase contrast velocity images at $i^{t h}$ frame, if available, provide the $\mathrm{x}$ and $y$-components of the instantaneous velocities for the mid-wall points. For all other points, we use the estimated velocity from the previous frames up to $j^{\text {th }}$ loop, $(i-1)^{t h}$ frame.

- The initial accelerations of all points are estimates from the previous frames up to $j^{\text {th }}$ loop, $(i-1)^{t h}$ frame.

- If $j=1$ and $i=1$, the initial Young's modulus and Poisson's ratio are set to 75000 Pascal and 0.47 respectively [12. Otherwise, we use the estimates from the previous frames up to $j^{t h}$ loop, $(i-1)^{t h}$ frame.

- The initial equivalent total loads are computed from the governing equations using the other initial condition.

Boundary conditions: the system equations are modified to account for the boundary conditions of the dynamic system. If the displacement of an arbitrary nodal point is known to be $U_{b}=b$, say from MR tagging images or shape-based boundary tracking [10], the constraint $k U_{b}=k b$ is added to the system governing equation, where $k$ is related to the confidence on the displacement.

Error measures of the estimation process: the filtering process is optimized by minimizing a set of error residuals, based on the differences between experimentally measured imaging and image-derived data, i.e. mid-wall MR phase contrast velocity and MR tagging/shape-tracked displacement, and those estimated by EKF framework. With our definition of state vector and incomplete image data, the matrix $D$ of Equation (4) should be properly chosen for all image frames, i.e. identity matrix where measurements are available and zero matrix for others.

After setting up all the initial and boundary conditions, the kinematics and material parameters can be estimated using the EKF strategy described earlier. 

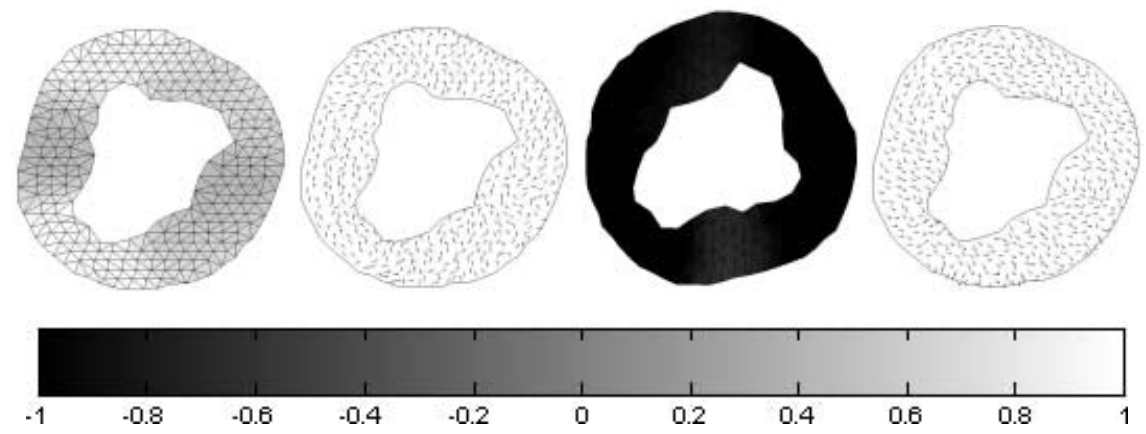

Fig. 4. Estimated maximum principle strain magnitude and direction maps (left) and minimum principle strain magnitude and direction maps (right) between ED and ES.

The estimation results at time $t$ will be used as input for time $t+1$. The estimation process needs to loop through all image frames in the cardiac cycle several times until convergence.

\section{Experiment}

Imaging/imaging-derived data: sixteen canine MR phase contrast velocity and magnitude images are acquired over the heart cycle, with imaging parameters flip angle $=30^{\circ}, T E=34 \mathrm{msec}, T R=34 \mathrm{msec}, F O V=28 \mathrm{~cm}, 5 \mathrm{~mm}$ skip 0, matrix $256 \mathrm{x} 128,4 \mathrm{nex}$, venc $=15 \mathrm{~cm} / \mathrm{sec}$. The image resolution is $1.09 \mathrm{~mm} / \mathrm{pixel}$, and the velocity intensity ranges from $-150 \mathrm{~mm} / \mathrm{sec}$ to $150 \mathrm{~mm} / \mathrm{sec}$, with the signs indicating the directions. Fig.1 shows the images at end-diastole. Endocardial and epicardial boundaries are extracted using velocity field constrained levelset strategy [11, and boundary displacements between consecutive frames are detected based on locating and matching geometric landmarks and a local coherent smoothness model [10].

Experimental results: the framework is implemented in Matlab, and all presented results are acquired after running through the image sequence six times, which takes about 40 minute on a Pentium 4 1.8GHZ computer. Fig. 2 shows the left ventricular meshes at end-diastole (ED) and end-systole (ES), as well as the displacement map between these two frames. Fig. 3 shows the x-, y- direction normal strain and shear strain distributions and mapping scale between ED and ES. The principle strains and their directions are shown in Fig.4. The final converged estimates of the Young's modulus and Poisson's ratio distributions and mapping scales are in Fig.5. Spatially, the Young's modulus varies from 72000 to 81000 Pascal, and the Poisson ratio varies from 0.40 to 0.50 . Analysis and interpretation of the results, as well as additional experiments, are underway.

\section{Conclusions}

We have developed a SFEM framework that can estimate left ventricular kinematics and material parameters simultaneously. We believe that this is the first 

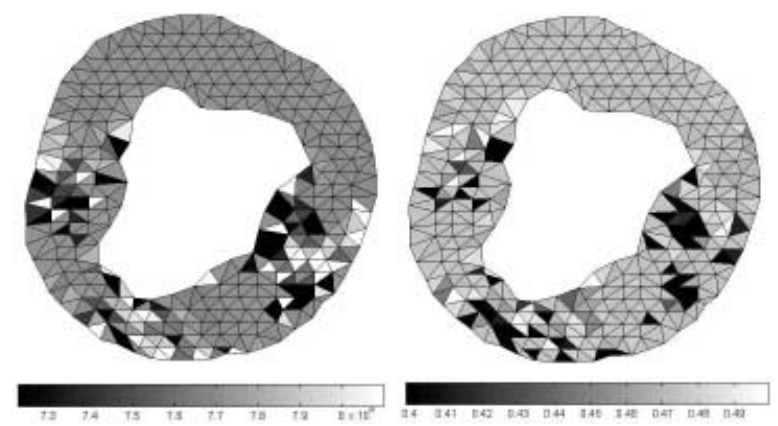

Fig. 5. Estimated Young's modulus (left) and Poisson's ratio (right) maps.

attempt in image analysis that incorporates uncertain constraining models in the ill-posed recovery problems, and the initial results are promising. We are working on issues related to robust estimation, numerical stability, realistic mechanical models, experiment validation, and extension to $3 \mathrm{D}$.

This work is supported in part by the Hong Kong CERG HKUST6057/00E, and by a HKUST Postdoctoral Fellowship Matching Fund.

\section{References}

1. Contreras, H.: The stochastic finite element method. Computer and Structure 12 (1980) 341-348

2. Frangi, A.J., Niessen, W.J., Viergever, M.A.: Three-dimensional modeling for functional analysis of cardiac images. IEEE Trans. Med. Imag. 20(1) (2001) 2-25

3. Glad T, T., Ljung, L.: Control Theory. Taylor \& Francis (2000) London

4. Hunter, P.J., Smaill, B.H.: The analysis of cardiac function: a continuum approach. Progress in Biophysics and Molecular Biology 52 (1989) 101-164

5. Ljung, L.: Asymptotic behavior of the extended Kalman filter as a parameter estimator for linear system. IEEE Trans. on Auto. Control AC24(1) (1979) 36-50

6. Moulton, M.J., Creswell, L.L., Actis, R.L., Myers, K.W., Vannier, M.W., Szabo, B.A., Pasque, M.K.: An inverse approach to determining myocardial material properties. Journal of Biomechanics 28(8) (1995) 935-948

7. Park, J., Metaxas, D.N., Axel, L.: Analysis of left ventricular wall motion based on volumetric deformable models and MRI-SPAMM. Medical Image Analysis 1(1) (1996) 53-71

8. Rao, S.K.: Comments on "Optimal guidance of proportional navigation". IEEE Transactions on Aerospace and Electronic systems 34(3) (1998) 981-982

9. Shi, P., Sinusas, A.J., Constable, R.T., Duncan, J.S.: Volumetric deformation analysis using mechanics-based data fusion: application in cardiac motion recovery. International Journal of Computer Vision 35(1) (1999) 87-107

10. Shi, P., Sinusas, A.J., Constable, R.T., Duncan, J.S.: Point-tracked quantitative analysis of left ventricular motion from 3D image sequences. IEEE Transactions on Medical Imaging 19(1) (2000) 36-50

11. Wong, L.N., Shi, P.: Velocity field constrained front propagation for segmentation of cardiac images. submitted to IEEE Workshop on Application of Computer Vision

12. Yamada, H.: Strength of Biological Material. Williams and Wilkins (1970) 